\title{
Effects of Weighted Hula-Hooping Compared to Walking on Abdominal Fat, Trunk Muscularity, and Metabolic Parameters in Overweight Subjects: A Randomized Controlled Study
}

\author{
Mari Lahelma ${ }^{a, b}$ Sanja Sädevirta ${ }^{a, b}$ Susanna Lallukka-Brück ${ }^{a, b}$ \\ Ksenia Sevastianova ${ }^{a}$ b Linda Mustelin $^{a}$, $b \quad$ Helena Gylling ${ }^{b}$ \\ Bonny Rockette-Wagner ${ }^{c}$ Andrea M. Kriska ${ }^{a, b}$ Hannele Yki-Järvinen ${ }^{a, b}$ \\ ${ }^{a}$ Minerva Foundation Institute for Medical Research, Helsinki, Finland; ${ }^{b}$ Department of \\ Medicine, Helsinki University Hospital, University of Helsinki, Helsinki, Finland; ' ${ }^{C}$ Department \\ of Epidemiology, University of Pittsburgh, Pittsburgh, PA, USA
}

\section{Keywords}

Body composition - Waist circumference - Obesity · Exercise

\begin{abstract}
Background: Weighted hula-hoops have gained popularity, but whether they indeed reshape the trunk or have beneficial metabolic effects in overweight subjects is unknown. Objectives: To determine effects of hula-hooping and walking matched for energy expenditure on android fat \%, trunk muscle mass, and metabolic parameters in a randomized cross-over study. Design: We recruited 55 overweight nondiabetic subjects, who were randomized to hulahooping (HULA) for 6 weeks using a 1.5-kg weighted hula-hoop followed by walking (WALK) for another 6 weeks or vice versa. The increments in energy expenditure were similar by HULA and WALK. Body composition (dual-energy X-ray absorptiometry) and metabolic parameters were measured at baseline and after HULA and WALK. The primary endpoint was the change in fat \% in the android region. Results: A total of 53 subjects (waist $92 \pm 1 \mathrm{~cm}$, body mass index $\left.28 \pm 1 \mathrm{~kg} / \mathrm{m}^{2}\right)$ completed the study. Body weight changed similarly $(-0.6 \pm 0.2 \mathrm{vs} .-0.5 \pm$ $0.2 \mathrm{~kg}$, nonsignificant; HULA vs. WALK). During the intervention the subjects hula-hooped on average $12.8 \pm 0.5 \mathrm{~min} /$ day and walked 9,986 \pm 376 steps/day. The $\%$ fat in the android region decreased significantly by HULA but not by WALK (between-group change $p<0.001$ ). Trunk muscle mass increased more by HULA than by WALK $(p<0.05)$. Waist circumference decreased more by HULA than by WALK $(-3.1 \pm 0.3 \mathrm{~cm}$ vs. $-0.7 \pm 0.4 \mathrm{~cm}, p<0.001$; HULA vs.
\end{abstract}

Mari Lahelma and Sanja Sädevirta contributed equally to this work. 
WALK). WALK but not HULA significantly lowered systolic blood pressure and increased HDL cholesterol while HULA significantly decreased LDL cholesterol. Conclusions: Hula-hooping with a weighted hula-hoop can be used to decrease abdominal fat \% and increase trunk muscle mass in overweight subjects. Its LDL lowering effect resembles that described for resistance training.

(C) 2019 The Author(s)

Published by S. Karger AG, Basel

\section{Introduction}

The metabolic/insulin resistance syndrome (MetS) refers to a cluster of abnormalities that are either causes or consequences of insulin resistance and coexist particularly in overweight sedentary subjects [1]. Physical activity reduces the risk of MetS in the face of minimal or no changes in body weight and it favorably influences all components of the MetS [2]. The WHO recommends physical activity for adults aged 18-64 years - at least 150 min of moderateintensity or 75 minutes of vigorous-intensity activity a week [3]. In addition, muscle-strengthening activities should be done involving major muscle groups 2 or more days a week. Walking is perhaps the most common form of physical activity, and often a suitable form of activity for overweight subjects and those with MetS. However, not everyone is motivated or able to walk and other factors such as the weather may pose limitations to walking. There is thus a need to search for and test alternative physical activities for such individuals.

Hula-hooping is an ancient type of dance, which has recently experienced a comeback in the form of aerobic core training [4]. Hula-hooping has been practiced in fitness classes worldwide and utilized in activity-promoting video games such as the Nintendo Wii. The energy expenditure (EE) of hooping has been estimated to vary from 3 to $7 \mathrm{kcal} / \mathrm{min}$ (moderate-intensity activity) depending on the hoop, hooping style, and individual metabolic factors [5]. The bigger and heavier the loop, the slower it rotates and the easier it is to use. Hula hooping activates muscles in the trunk such as lower abdominals, psoas major, and back extensors as well as those in the lower limbs (hip abductors and, to a small extent, muscles of the knee and ankle) [6]. Data on the effects of hula-hooping on body composition are limited to one uncontrolled study, in which hula-hooping decreased waist circumference [7]. However, in this study, metabolic parameters or fat and muscle masses were not determined. It is unknown whether hula-hooping changes metabolic parameters and, if so, whether such changes resemble those associated with resistance or aerobic training.

In the present study we wished to compare the effects of hula-hooping (HULA) and walking (WALK) in overweight subjects. Our hypothesis was that HULA decreases abdominal fat $\%$ and increases trunk muscle mass more than WALK. The primary endpoint was the $\%$ change in android fat as measured by dual-energy X-ray absorptiometry (DEXA). Secondarily, we examined whether HULA and WALK have different effects on metabolic features such as glucose, insulin, blood pressure, and lipids. To this end, we randomized 55 volunteers to either HULA or WALK for two 6-week periods in a cross-over fashion. Key parameters from the first period of intervention, where the subjects were randomized to either HULA or WALK, were also analyzed as if the trial was performed using a parallel design.

\section{Materials and Methods}

\section{Study Subjects}

The study subjects were recruited through advertisements using intranet and bulletin boards in the Hospital District of Helsinki and Uusimaa. The following inclusion criteria were 


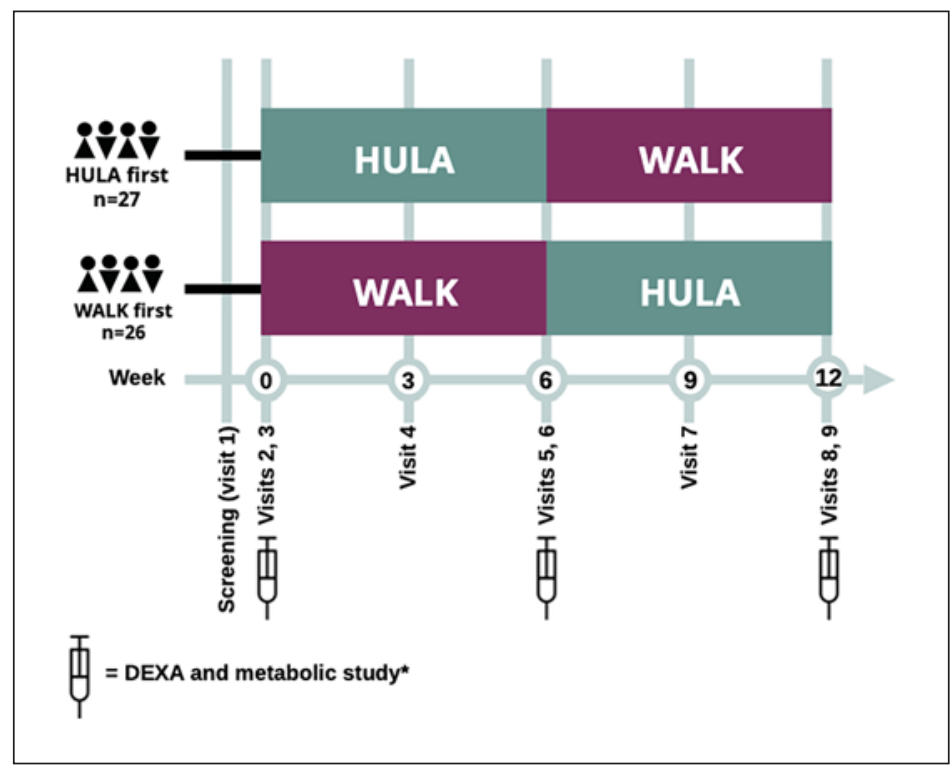

Fig. 1. Flow chart of study design. A total of 55 subjects were recruited and randomized into two groups. Half of the subjects started with 6 weeks of hula-hooping (HULA) followed by 6 weeks of walking (WALK), while the other half started by walking for 6 weeks and then switched to hula-hooping for another 6 weeks. A total of 53 subjects completed the study. The primary endpoint was the \% change in android fat as measured by dual-energy X-ray absorptiometry (DEXA) before and after the interventions measured at visits 3 (week 0), 6 (week 6), and 9 (week 12). * The metabolic study (visits 2, 5, and 8) included blood sampling for measurement of features of the metabolic/insulin resistance syndrome (lipids, liver enzymes, glucose, $\mathrm{HbA}_{1 \mathrm{c}}$, and insulin) in addition to recording of blood pressure, body weight, height, and waist and hip circumferences.

applied: (i) age between 18 and 70 years, (ii) central adiposity as measured by waist circumference ( $>80 \mathrm{~cm}$ in females and $>94 \mathrm{~cm}$ in males) [8], (iii) ability to hula-hoop for a minimum of 1 min and possibility to hula-hoop either at home or at work, (iv) ability to communicate meaningfully with the investigator, and (v) legal competence to provide written informed consent. Exclusion criteria included: (i) clinical or biochemical evidence of diseases other than obesity as judged by history, physical examination, and standard laboratory tests (vide infra), (ii) excessive use of alcohol, i.e., over $20 \mathrm{~g}$ /day, (iii) use of medications known to affect glucose or lipid metabolism, and (iv) pregnancy or lactation.

\section{Study Objectives}

The primary objective was to evaluate the \% change in fat in the android region (vide infra) as measure by DEXA during 6 weeks of HULA compared to 6 weeks of WALK. Secondary objectives included comparison of changes induced by HULA compared to WALK in waist circumference, relative and absolute trunk muscle mass measured by DEXA, and metabolic parameters including fasting plasma glucose, fasting serum insulin, fasting serum lipids, and liver enzymes.

\section{Study Design}

An overview of the study design and visits is shown in Figure 1. The study had a crossover design in which half of the subjects $(n=27)$ first hula-hooped for 6 weeks and then walked for 6 weeks, while the other half $(n=26)$ first walked for 6 weeks and then hulahooped for 6 weeks. Key parameters were also analyzed using a parallel design, i.e., by 
Lahelma et al.: Hula-Hooping

comparing groups randomized to hula-hooping (HULA first) or walking (WALK first) for the first 6 weeks. To match EEs of the groups, we measured the effects of weighted hula-hooping and walking on heart rate (HR) in 10 volunteers (age $38 \pm 2$ years, body mass index $25 \pm 1$ $\mathrm{kg} / \mathrm{m}^{2}$ ) and then estimated average EEs. Resting HR averaged $64 \pm 1 \mathrm{bpm}$. HR for $10 \mathrm{~min}$ averaged $95 \pm 2 \mathrm{bpm}$ during hula-hooping and $97 \pm 3 \mathrm{bpm}$ during walking. The EE of hulahooping and walking were then calculated using an equation predicting EE based on HR, weight, age, and gender [9]. EE averaged $3.8 \pm 0.2 \mathrm{kcal} / \mathrm{min}$ during hula-hooping and $4.0 \pm 0.3$ $\mathrm{kcal} /$ min during walking. We therefore recommended the WALK group to walk an extra 10 min/day and the HULA group to HULA $11 \mathrm{~min} /$ day to achieve similar EEs (approximately 41 $\mathrm{kcal} /$ day) by both activities.

Screening (Visit 1). Subjects interested and potentially eligible for the study were invited for a screening visit after a 12-h fast. All the visits occurred at the clinical research unit of the Helsinki University Hospital. The subjects attended visits individually. At the screening visit, written informed consent was obtained and a history and physical examination, including measurement of waist and hip circumferences, body weight, and height, and recording of an electrocardiogram, were performed. Blood samples (complete blood counts, and concentrations of creatinine, sodium, potassium, alanine aminotransferase [ALT], glucose, and thyroidstimulating hormone) were taken. A pregnancy test was performed in women of child-bearing potential. The ability to hula-hoop for at least $1 \mathrm{~min}$ without dropping the hula-hoop was tested for. Eligible subjects were randomized to start either with HULA or WALK. Of a total of 61 subjects who were screened, 55 were eligible for the study; 6 subjects were not eligible because of abnormal laboratory test results at screening $(n=6)$, and 2 subjects dropped out from the study (traumatic fracture unrelated to the study in one case, and lack of compliance in the other). A total of 53 subjects completed the study.

Baseline Measurements (Visits 2 and 3). Subjects eligible for the study based on the screening visit were invited to a baseline visit, during which recording of blood pressure, body weight, height, and waist and hip circumferences was repeated. Baseline blood samples were taken after an overnight fast for measurement of complete blood count and concentrations of ALT, HDL and LDL cholesterol, triglycerides, glucose, glycosylated hemoglobin $A_{1 c}$ $\left(\mathrm{HbA}_{1 \mathrm{c}}\right.$ ), and insulin (visit 2). Baseline body composition was measured by DEXA within a few days from the baseline study visit (visit 3).

HULA. After visit 3, the subjects commenced 6 weeks of either hula-hooping or walking (Fig. 1). The exercise protocol consisted of $6 \mathrm{~min}$ of hula-hooping per day for the first week with an addition of 2 min per day every week for the HULA group. All subjects were offered a 1-h teaching session within 1 week prior to commencement of the HULA intervention (Fig. 2). During this session, the subjects were taught the technique of hula-hooping (A.S.). Each subject was provided with a hula-hoop weighing $1.5 \mathrm{~kg}$.

WALK. The study subjects were provided with a pedometer (Walking Style III; Omron Healthcare Corporation Ltd., Kyoto, Japan) which was worn to monitor activity during both the HULA and WALK periods. In addition, the subjects kept a diary to record the number of minutes hula-hooped each day. The study subjects were instructed to continue their normal diet and maintain their other exercise habits as prior to the study.

Measurements during First Exercise Intervention (Visit 4). The subjects attended a clinical visit in the middle of the exercise intervention (end of week 3). At this visit, body weight, waist and hip circumferences, and blood pressure were measured, and compliance to the exercise protocol was reviewed based on the exercise diary that the study subjects were asked to keep.

Measurements after First Exercise Intervention (Visits 5 and 6). Upon completion of 6 weeks of HULA or WALK, the subjects were invited to visits 5 and 6 , which were identical to those of visits 2 and 3 described above. 
Fig. 2. A study subject hula-hooping using a 1.5 -kg weighted hulahoop (published with permission of the subject).

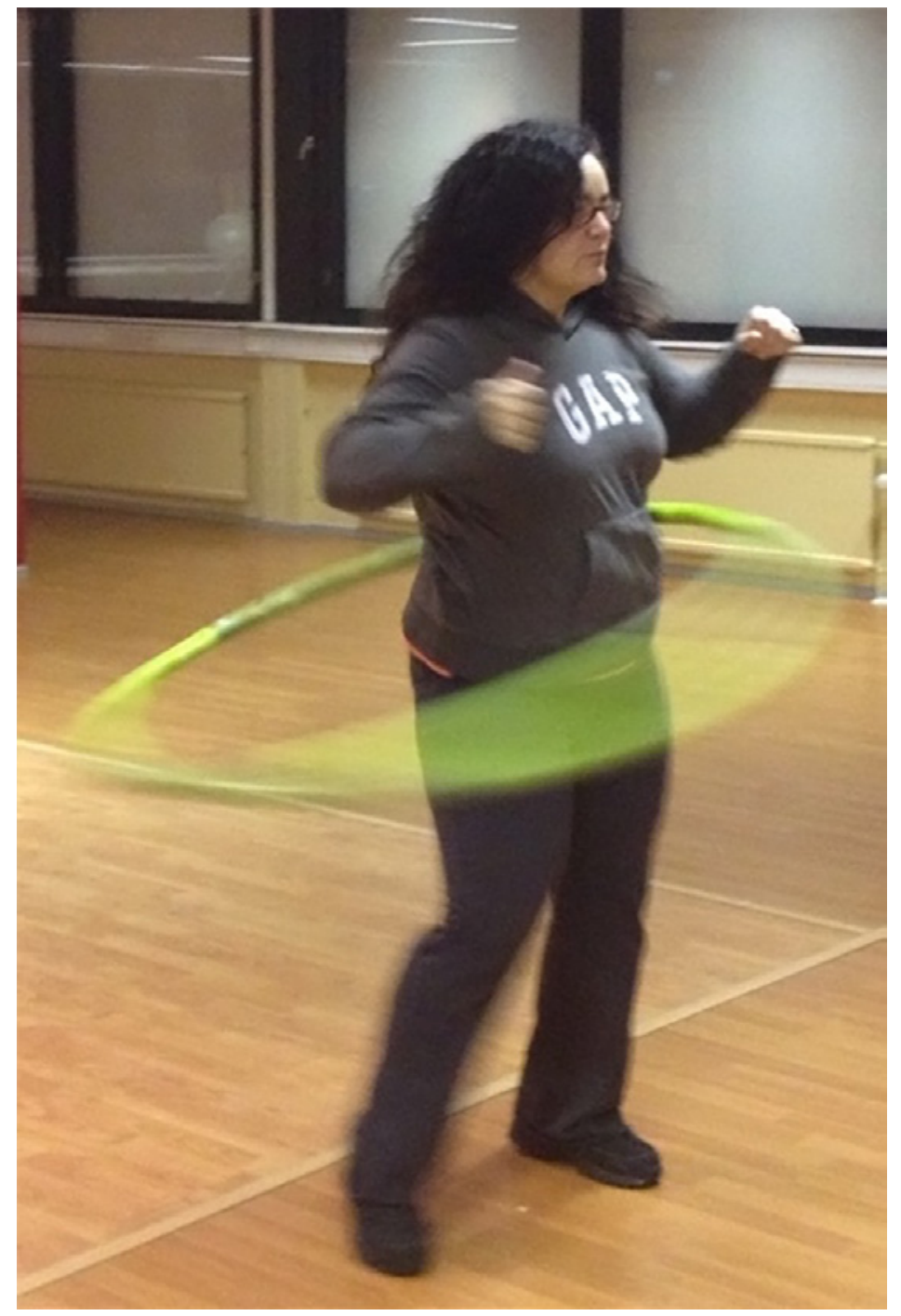

Cross-Over of Exercise Intervention. After visits 5 and 6, the study subjects crossed over to hula-hooping for those walking initially and vice versa. A hula-hooping training session was held for those commencing HULA after WALK.

Measurements during Second Exercise Intervention (Visit 7). A clinical visit in the middle of the exercise intervention period was carried out as described for visit 4.

Measurements after Second Exercise Intervention (Visits 8 and 9). The final visits were carried out upon completion of 6 weeks of the second exercise intervention. The protocols of these visits were identical to those of visits 5 and 6.

\section{Measurements of Body Composition}

DEXA and Other Measures of Body Composition. Body composition variables were measured and calculated automatically by DEXA and its integral commercial software (Lunar Prodigy Advance; GE Healthcare, Madison, WI, USA). The android region includes an area from the top of the iliac crest to $20 \%$ of the distance from the iliac crest to the bottom of the subject's head. The gynoid region extends from the top of the greater trochanter down a distance twice the height of the android region. The trunk region includes the neck, chest, 
Lahelma et al.: Hula-Hooping

abdominal, and pelvic areas. Its upper border is the chin and its lower border the intersect between the middle of the femoral necks without touching the brim of pelvis [10]. The $\%$ fat values in a given region were calculated as $\%$ fat $=$ fat mass $/($ fat mass + lean mass + bone mass) $\times 100$. The $\%$ lean mass values were calculated as $\%$ lean $=$ lean mass $/$ (fat mass + lean mass + bone mass) $\times 100$. Body weight, height, and waist and hip circumferences were measured as previously described [8].

\section{Analytical Procedures}

Fasting glucose, $\mathrm{HbA}_{1 \mathrm{c}}$, insulin, LDL and HDL cholesterol, triglyceride concentrations, and ALT were measured as previously described [11]. To estimate whether liver fat content changed during the interventions, we calculated the \% liver fat using an equation, which was developed in our laboratory by quantifying liver fat by proton magnetic resonance spectroscopy $\left({ }^{1} \mathrm{H}-\mathrm{MRS}\right)$ in 470 subjects [8].

Since we observed a significant decrease in LDL cholesterol (see Results) during the HULA but not the WALK period, we determined whether this change was associated with markers of cholesterol absorption or synthesis. For this, concentrations of squalene and noncholesterol sterols were measured from nonsaponifiable serum material with capillary gas-liquid chromatography using a 50-m-long Ultra 1 capillary column (Agilent Technologies, Wilmington, DE, USA) [12].

\section{Statistical Methods}

All data were tested for normality of distribution using the D'Agostino-Pearson normality test. Normally distributed data are shown as means \pm standard error of mean (SEM) and nonnormally distributed data are shown as median (followed by the 25 and 75 percentiles). Baseline characteristics were compared using the unpaired $t$ test for normally distributed data and the Mann-Whitney test for non-normally distributed data. Intervention effects between intervention groups (HULA vs. WALK group) were analyzed using the clustered complex samples general linear model with change (before vs. after intervention values) as the dependent variable, intervention group (HULA vs. WALK) as the fixed factor, and baseline values and period (order of intervention) as covariates (see online suppl. Table 4; for all online suppl. material, see www.karger.com/doi/10.1159/000500572). Key variables were also analyzed using a parallel design by comparing groups randomized to hula-hooping or walking during the first 6-week period (HULA first vs. WALK first). For this, changes (the group $\times$ time interaction) were compared using repeated-measures 2-way ANOVA with intervention group (HULA vs. WALK) as the grouping variable and time (before vs. after) as the paired factor. The calculations were performed using GraphPad Prism version 7.0 for Mac (GraphPad Software Inc., San Diego, CA, USA) and IBM SPSS Statistics 24 (IBM corporation, Armonk, NY, USA).

\section{Results}

\section{Baseline Characteristics}

Physical and biochemical characteristics of all subjects before the HULA $(n=53)$ and the WALK $(n=53)$ periods were comparable (Table 1$)$. The subjects were mostly women $(n=50)$. For analysis of the data using a parallel design, online supplementary Table 1 shows characteristics of the subjects randomized first to hula-hooping (HULA first, $n=27$ ) or walking (WALK first, $n=26$ ) for the first 6 weeks. These groups were also comparable with respect to physical and biochemical characteristics prior to receiving any intervention. 


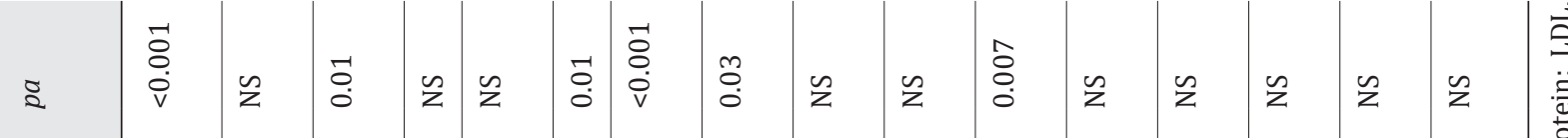

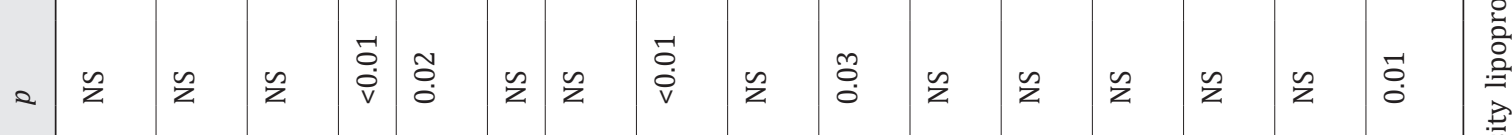

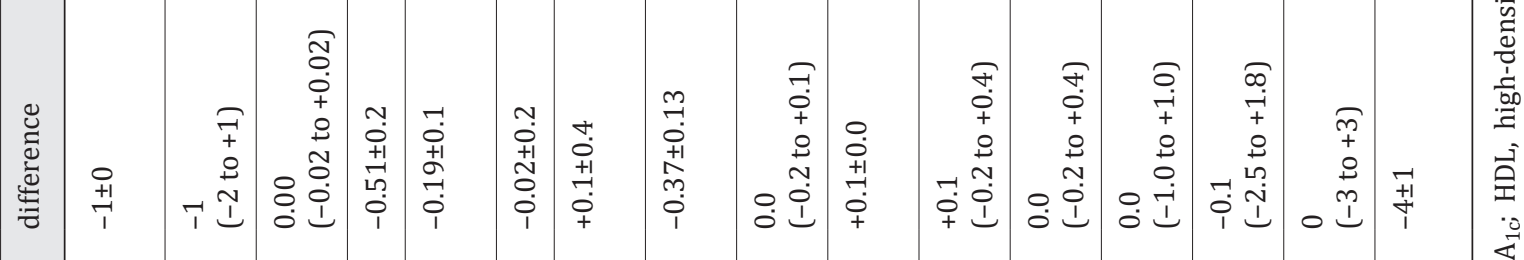

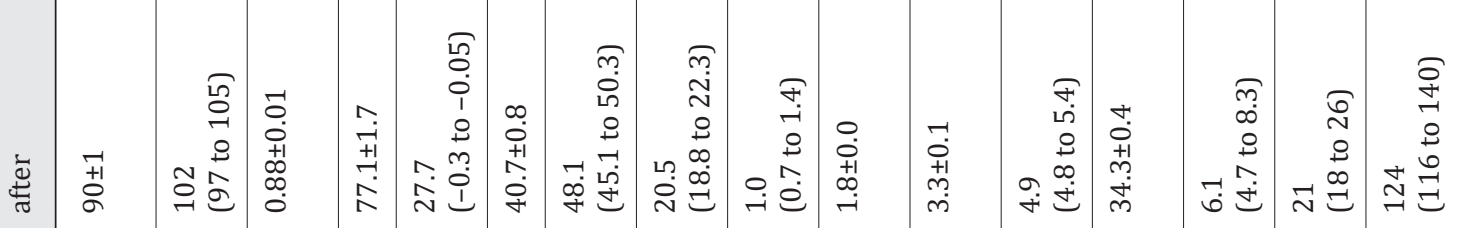

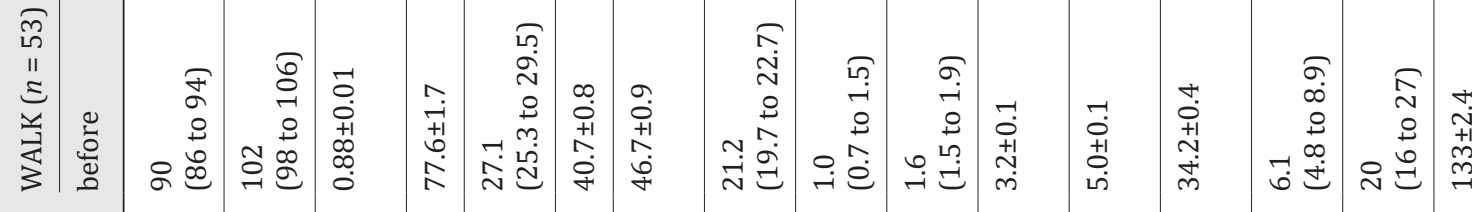

离

ह

(2)

$\frac{0}{\frac{0}{0}}$

$\frac{\sqrt{2}}{2}$

एี

幽

๘

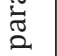

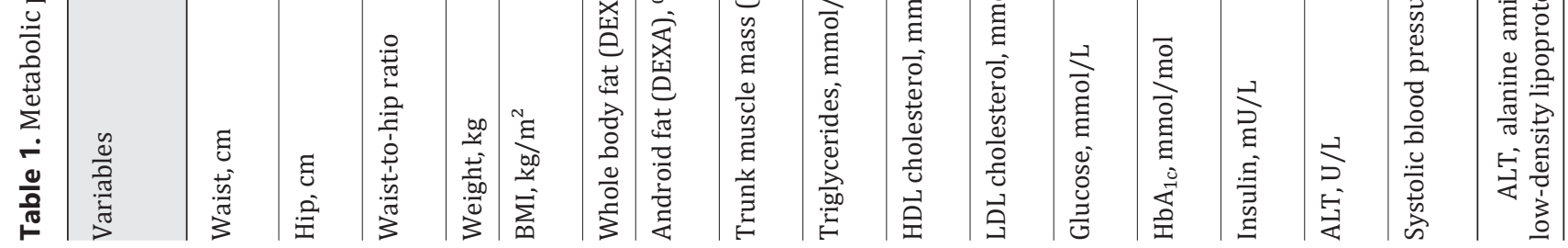

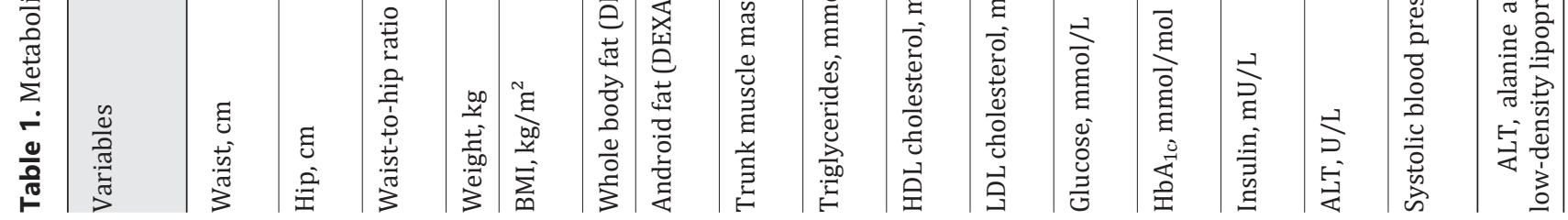

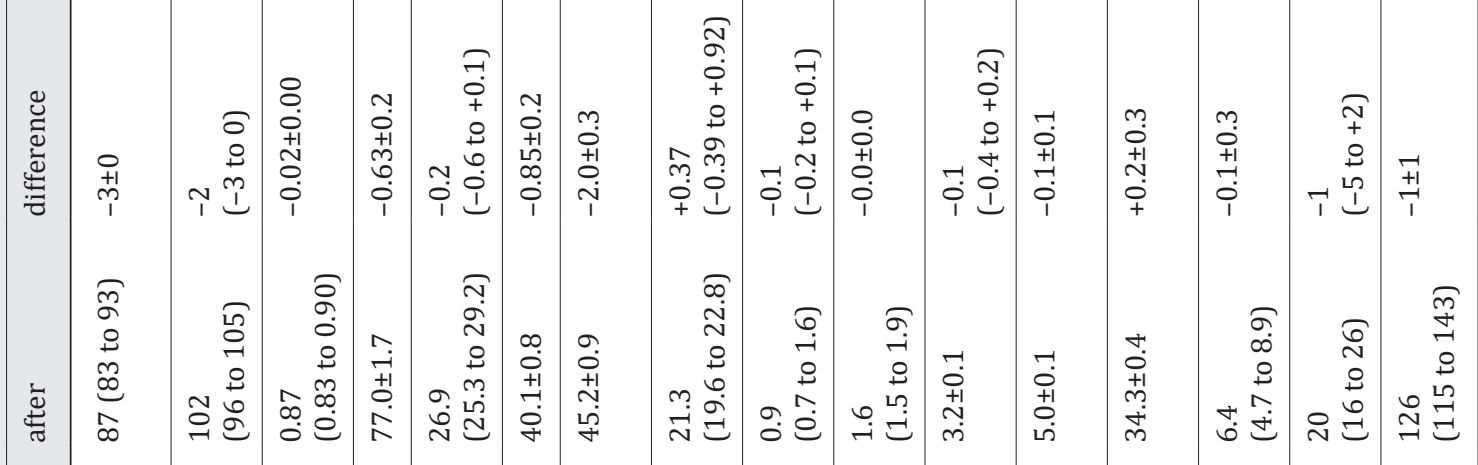
\&

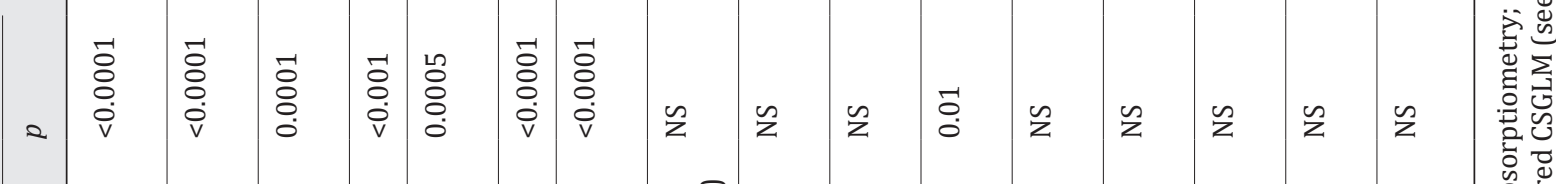

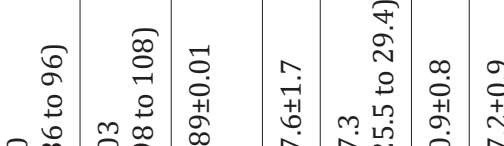


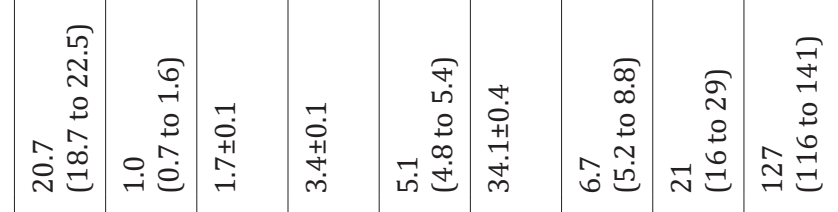




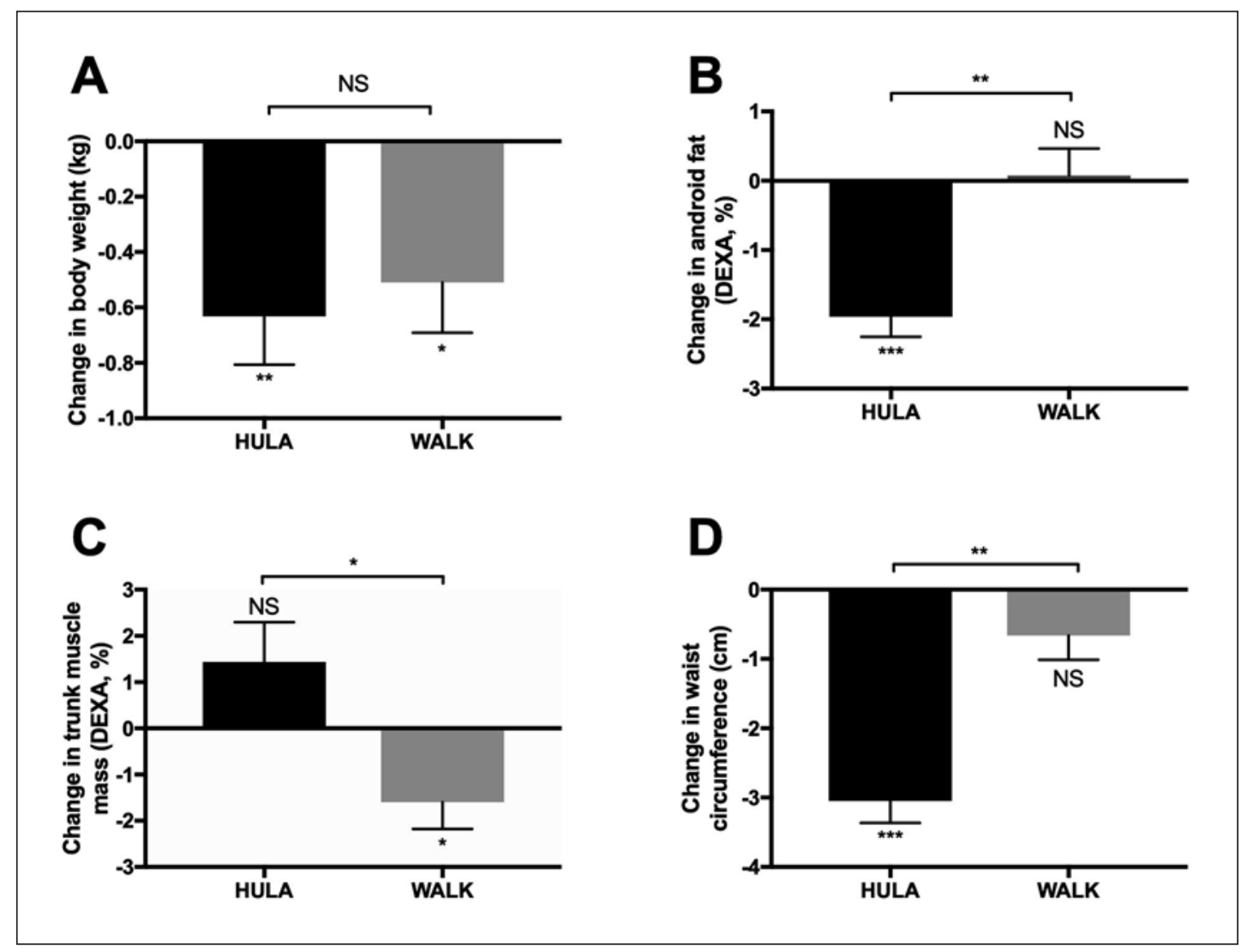

Fig. 3. Effect of exercise modalities on body weight (A), android fat $\%$ (B), trunk muscle mass (C), and waist circumference (D). Data are given as mean \pm SEM. ${ }^{*} p<0.05 ;{ }^{* *} p<0.001$; ${ }^{* *} p<0.0001$; NS, nonsignificant.

\section{Compliance}

During the WALK period, the subjects reported the steps taken during $94 \pm 2 \%$ of all days, and during the HULA period during $92 \pm 2 \%$ of all days. The subjects hula-hooped on average $12.8 \pm 0.5 \mathrm{~min} /$ day. During the WALK period, the subjects walked on average 9,986 \pm 376 steps/day and during the HULA period 8,974 \pm 359 steps/day (online suppl. Table 2).

Body Weight, Android Fat \%, and Other Measures of Body Composition

Cross-Over Design. Changes in body weight were comparable between the exercise modalities $(-0.6 \pm 0.2$ vs. $-0.5 \pm 0.2 \mathrm{~kg}$, NS; change during HULA vs. WALK; Fig. $3 \mathrm{~A})$. The primary endpoint, the android fat $\%$, decreased significantly $(p<0.001$ with period and before-intervention value as covariates) during HULA $(-2.0 \pm 0.3 \%, p<0.0001)$ but not during WALK $(0.1 \pm 0.4 \%$; Fig. $3 \mathrm{~B})$. The absolute trunk muscle mass increased significantly more ( $p<0.03$ with period and before-intervention value as covariates) during HULA than during WALK (Fig. 3C). Waist circumference decreased significantly more ( $p<0.001$ with period and before-intervention value as covariates) by HULA $(-3.1 \pm 0.3 \mathrm{~cm})$ than WALK $(-0.7 \pm 0.4 \mathrm{~cm}$; Fig. 3D $)$.

Pre- and postmenopausal women have differences in their fat distribution [13]. The decreases in abdominal fat $\%(-2.2 \pm 0.4$ vs. $-1.7 \pm 0.5 \%$, NS; pre- vs. postmenopausal) and waist circumference ( $-3.0 \pm 0.4$ vs. $-3.1 \pm 0.5 \mathrm{~cm}$, NS; pre- vs. postmenopausal) were similar 
Lahelma et al.: Hula-Hooping

in pre- and postmenopausal women during HULA, but the study was not powered to examine the effect of hula-hooping based on menopausal status.

Parallel Design. When analyzed using data from the HULA-first and WALK-first groups, waist circumference decreased significantly more $(p=0.02$ for changes in repeatedmeasures 2-way ANOVA) in the HULA-first $(-3.1 \pm 0.4 \mathrm{~cm})$ than the WALK-first $(-1.0[-2.6$ to 0.5 ] $\mathrm{cm}$ ) group. Changes in other body composition parameters were comparable (online suppl. Table 1).

\section{Metabolic Parameters}

Cross-Over Design. LDL cholesterol decreased significantly more ( $p=0.007$ for intervention with before-intervention LDL and period as covariates) during HULA ( -0.1 [ -0.40 to $+0.2] \mathrm{mmol} / \mathrm{L})$ than WALK $(+0.1$ [ -0.2 to 0.4$] \mathrm{mmol} / \mathrm{L}, \mathrm{NS})$. The period effect was not significant, but LDL cholesterol decreased more in subjects with elevated before-intervention LDL cholesterol ( $p=0.007$; online suppl. Table 4). HDL cholesterol remained unchanged during HULA $(0.0 \pm 0.0 \mathrm{mmol} / \mathrm{l})$ but increased $(p<0.05)$ significantly during WALK $(+0.1 \pm 0.0$ $\mathrm{mmol} / \mathrm{L})$. The period effect was not significant, but HDL increased more in subjects with initially low HDL cholesterol ( $p<0.0001$ for before-intervention HDL as covariate). There were no statistically significant differences in changes in serum triglycerides between the groups (Table 1). Systolic blood pressure remained unchanged during HULA $(-1 \pm 1 \mathrm{~mm} \mathrm{Hg}$, NS; before vs. after HULA) but decreased significantly during WALK $(-4 \pm 1 \mathrm{~mm} \mathrm{Hg}, p<0.02$; before vs. after WALK). Diastolic blood pressure did not change in either group. There were no significant differences in changes between the groups in glucose, $\mathrm{HbA}_{1 c}$, insulin, or ALT concentrations (Table 1). For $p$ values for covariates see online supplementary Table 4. Since there was a significant difference between the groups in the change in LDL cholesterol concentrations, circulating markers of cholesterol synthesis and absorption were measured with GC-MS. Markers of synthesis or absorption did not change significantly during HULA or WALK (online suppl. Table 3).

Parallel Design. LDL decreased significantly more $(\mathrm{p}=0.002)$ in the HULA-first than the WALK-first group. There were no significant differences in other metabolic parameters (online suppl. Table 1).

\section{Discussion/Conclusion}

There are no controlled studies addressing the effects of hula-hooping with weighted hula-hoops on body composition or metabolic parameters. We found in a group of overweight subjects that 6 weeks of hula-hooping for an average duration of 13 min per day significantly decreased waist circumference and body fat in the android region and increased trunk muscularity compared to a period of walking. These results were not due to changes in body weight as hula-hooping and walking induced trivial and similar weight loss. Walking but not hulahooping increased HDL cholesterol and decreased systolic blood pressure while hula-hooping lowered LDL cholesterol.

Hula-hooping decreased waist circumference by an amount that has been associated with changes in other components of the MetS in studies using weight loss and aerobic training as the therapeutic intervention [14]. In the meta-analysis of Yamaoka and Tango [14], a decrease in waist circumference $(-2.7 \mathrm{~cm})$, i.e., an amount similar to that attained in our study $(-3 \mathrm{~cm})$, was associated with significant improvements in metabolic parameters including decreases in systolic $(-6.4 \mathrm{~mm} \mathrm{Hg})$ and diastolic $(-3.3 \mathrm{~mm} \mathrm{Hg})$ blood pressure, serum triglycerides $(-0.14 \mathrm{mmol} / \mathrm{L})$, and fasting blood glucose $(-0.63 \mathrm{mmol} / \mathrm{L})$. However, correlation does not prove causality $[15,16]$, and it is indeed controversial whether the asso- 
Lahelma et al.: Hula-Hooping

ciation between abdominal obesity and insulin resistance is "causative or correlative" [15] or "a major culprit or simply an innocent bystander" [16]. The present data show that hulahooping locally reshapes the waist without changing other components of the MetS. This effect is reminiscent of the effect of abdominal liposuction on whole-body insulin sensitivity, blood pressure, or lipids [17]. In the latter study, removal of $44 \%$ of subcutaneous adipose tissue in 15 women had no effect on whole body insulin sensitivity [17].

Regarding changes in markers of cardiovascular risk, WALK increased HDL cholesterol and decreased systolic blood pressure. These changes are in keeping with known beneficial effects of aerobic training on blood pressure and lipids $[18,19]$. Our patients were already active as they attained the global health recommendations of walking time at baseline. By design, the extra walking time was low (10 $\mathrm{min} /$ day $)$ and may underestimate the true benefits of walking. Nevertheless, systemic changes with potential long-term cardiovascular benefits were observed. This reinforces the idea that even moderately active people can attain health benefits with a little increase in activity such as walking [20]. Given that WALK and HULA induced similar increases in EEs, as determined by changes in HR, one might not expect these activities to differentially affect cardiovascular risk factors. However, data comparing two different types of exercise in the face of equal increases in HR are sparse. There are, indeed, several examples where maximal aerobic capacity $\left(\mathrm{VO}_{2} \mathrm{max}\right)$ and cardiovascular risk factors have not changed in parallel during physical training. For example, in 8 out of 11 studies examining the effects of aerobic exercise such as walking or bicycling on markers of cardiovascular risk, beneficial changes in serum triglycerides or HDL cholesterol were observed in the absence of an improvement in $\mathrm{VO}_{2}$ max or change in body weight [21].

HULA but not WALK significantly decreased LDL cholesterol but did not change HDL cholesterol or triglycerides. The decrease in LDL cholesterol and increase in trunk muscle mass resemble effects observed during resistance training [22-25]. According to metaanalyses by Kelley et al. [22-24] $(2004,2006,2009)$, resistance training and aerobic training have slightly different effects on lipid profile, as resistance training decreases LDL cholesterol and non-HDL cholesterol without significant effects on HDL cholesterol, while aerobic training primarily increases HDL cholesterol and, to a lesser degree, decreases LDL. The difference in LDL cholesterol concentrations between the groups in the present study could be due to changes in cholesterol absorption or synthesis, or perhaps due to changes in adipose tissue, which is one of the largest body cholesterol pools in humans [26]. A dietary explanation is unlikely since the order of the two exercise periods was randomized. As we perceived the distinct effects on lipid profile as fascinating, we further investigated the markers of cholesterol synthesis (desmosterol, lathosterol, cholestenol, and squalene) and absorption (cholestanol, campesterol, sitosterol, and avenasterol) (online suppl. Table 3). There were no significant changes between groups. The mechanism underlying the decrease in LDL cholesterol thus remains unclear. Possibly, changes in adipose tissue cholesterol stores as a result of body reshaping might influence circulating LDL cholesterol but this remains speculative. On the other hand, it is still equally unclear why resistance training decreases LDL cholesterol. The greater decrease in abdominal fat \% by hula-hooping compared to walking could also differentially affect concentrations of adipokines, which may regulate low-grade inflammation and cardiovascular risk [27, 28].

Cross-over trials offer advantages in terms of power since each subject is studied repeatedly. On the other hand, disentangling treatment effects from time and carryover effects can be challenging [29]. These were not issues in the present study since comparison of the main treatment effects for waist circumference and LDL cholesterol using parallel design yielded similar results to those in the analysis of differences from the cross-over data. Physical activity of the subjects was high, as it exceeded the global recommendation of daily physical activity by WHO [3]. Whether hula-hooping would have even more beneficial effects 
Lahelma et al.: Hula-Hooping

in less active individuals with more severe features of MetS remains to be established. Men were less eager to volunteer for the present study and therefore we cannot really make conclusions regarding the effects of hula-hooping in men. Also, the results may not be applicable to severely obese subjects.

We conclude that hula-hooping can reshape the body by increasing abdominal muscle mass and decreasing waist circumference, which can be helpful extrinsic motivators to exercise for overweight individuals. Interestingly, hula-hooping decreased LDL cholesterol, a change typically induced by resistance rather than aerobic training. In contrast, even a small increase in walking in already active overweight people had positive effects on lipids and blood pressure. Taken together these data suggest that hula-hooping may complement the beneficial effects of aerobic activities, such as walking, and could therefore be included among activities recommended for overweight individuals.

\section{Statement of Ethics}

The study protocol was approved by the Medical Ethics Committee of the Hospital District of Helsinki and Uusimaa. The study is registered at www.clinicaltrials.gov (No. NCT01913171). The subjects gave their written informed consent.

\section{Disclosure Statement}

The authors have no conflicts of interest to declare.

\section{Funding Sources}

This study was supported by research grants from EVO and Sigrid Juselius Foundations.

\section{Author Contributions}

H.Y.-J.: study concept and design. S.S., L.M., and K.D.: acquisition of clinical data. M.L., S.S., A.M.K., B.R.-W., and S.L.-B.: analysis and interpretation of data and writing of the manuscript. H.G.: acquisition of sterol data. A.S.: HULA training session. All authors performed critical revision of the manuscript for important intellectual content.

\section{References}

1 Yki-Järvinen H. Non-alcoholic fatty liver disease as a cause and a consequence of metabolic syndrome. Lancet Diabetes Endocrinol. 2014 Nov;2(11):901-10. Available from: http://www.thelancet.com/journals/landia/ article/PIIS2213-8587(14)70032-4/abstract.

2 Ross R, Janiszewski PM. Is weight loss the optimal target for obesity-related cardiovascular disease risk reduction? Can J Cardiol. 2008;24 Suppl D:25D-31D.

3 World Health Organization. Global recommendations on physical activity for health [cited 2018 Mar 12]. Geneva:World Health Organization; 2010.Available from: http://www.ncbi.nlm.nih.gov/books/NBK305057/.

4 HulaHooping.com. Hoop history: Learn about hooping, hula hoops, evolution of hooping [cited 2018 Mar 12]. Available from: http://www.hulahooping.com/history.html.

5 ACE-sponsored research. Hooping-Effective workout or child's play? [cited 2018 Jan 11]. Available from: https://www.acefitness.org/certifiednewsarticle/1094/ace-sponsored-research-hooping-effectiveworkout. 
Lahelma et al.: Hula-Hooping

6 Cluff T, Robertson DG, Balasubramaniam R. Kinetics of hula hooping: an inverse dynamics analysis. Hum Mov Sci. 2008 Aug;27(4):622-35.

7 McGill SM, Cambridge ED, Andersen JT. A six-week trial of hula hooping using a weighted hoop: effects on skinfold, girths, weight, and torso muscle endurance. J Strength Cond Res. 2015 May;29(5):1279-84.

8 Kotronen A, Peltonen M, Hakkarainen A, Sevastianova K, Bergholm R, Johansson LM, et al. Prediction of nonalcoholic fatty liver disease and liver fat using metabolic and genetic factors. Gastroenterology. 2009 Sep; 137(3):865-72.

9 Keytel LR, Goedecke JH, Noakes TD, Hiiloskorpi H, Laukkanen R, van der Merwe L, et al. Prediction of energy expenditure from heart rate monitoring during submaximal exercise. J Sports Sci. 2005 Mar;23(3):289-97.

10 Mazess RB, Barden HS, Bisek JP, Hanson J. Dual-energy x-ray absorptiometry for total-body and regional bonemineral and soft-tissue composition. Am J Clin Nutr. 1990 Jun;51(6):1106-12.

11 Sevastianova K, Kotronen A, Gastaldelli A, Perttilä J, Hakkarainen A, Lundbom J, et al. Genetic variation in PNPLA3 (adiponutrin) confers sensitivity to weight loss-induced decrease in liver fat in humans. Am J Clin Nutr. 2011 Jul;94(1):104-11.

12 Simonen P, Kotronen A, Hallikainen M, Sevastianova K, Makkonen J, Hakkarainen A, et al. Cholesterol synthesis is increased and absorption decreased in non-alcoholic fatty liver disease independent of obesity. J Hepatol. 2011 Jan;54(1):153-9.

13 Zamboni M, Armellini F, Milani MP, De Marchi M, Todesco T, Robbi R, et al. Body fat distribution in pre- and post-menopausal women: metabolic and anthropometric variables and their inter-relationships. Int J Obes Relat Metab Disord. 1992 Jul;16(7):495-504.

14 Yamaoka K, Tango T. Effects of lifestyle modification on metabolic syndrome: a systematic review and metaanalysis. BMC Med. 2012 Nov;10(1):138.

15 Frayn KN. Visceral fat and insulin resistance-causative or correlative? Br J Nutr. 2000 Mar;83(S1 Suppl 1):S71-7.

16 Seidell JC, Bouchard C. Visceral fat in relation to health: is it a major culprit or simply an innocent bystander? Int J Obes Relat Metab Disord. 1997 Aug;21(8):626-31.

17 Klein S, Fontana L, Young VL, Coggan AR, Kilo C, Patterson BW, et al. Absence of an effect of liposuction on insulin action and risk factors for coronary heart disease. N Engl J Med. 2004 Jun;350(25):2549-57.

18 Wang Y, Xu D. Effects of aerobic exercise on lipids and lipoproteins. Lipids Health Dis. 2017 Jul;16(1):132.

19 Mann S, Beedie C, Jimenez A. Differential effects of aerobic exercise, resistance training and combined exercise modalities on cholesterol and the lipid profile: review, synthesis and recommendations. Sports Med. 2014 Feb;44(2):211-21.

20 Blair SN, Kohl HW, Gordon NF, Paffenbarger RS Jr. How much physical activity is good for health? Annu Rev Public Health. 1992;13(1):99-126.

21 Textbook of sports medicine: Basic science and clinical aspects of sports injury and physical activity [cited 2018 Mar 22]. Available from: https://www.wiley.com/en-us/Textbook+of+Sports+Medicine\%3A+Basic+Sc ience+and+Clinical+Aspects+of+Sports+Injury+and+Physical+Activity-p-9780632065097.

22 Kelley GA, Kelley KS. Impact of progressive resistance training on lipids and lipoproteins in adults: a metaanalysis of randomized controlled trials. Prev Med. 2009 Jan;48(1):9-19.

23 Kelley GA, Kelley KS. Aerobic exercise and lipids and lipoproteins in men: a meta-analysis of randomized controlled trials. J Mens Health Gend. 2006;3(1):61-70.

24 Kelley GA, Kelley KS, Tran ZV. Aerobic exercise and lipids and lipoproteins in women: a meta-analysis of randomized controlled trials. J Womens Health (Larchmt). 2004 Dec;13(10):1148-64.

25 Willis LH, Slentz CA, Bateman LA, Shields AT, Piner LW, Bales CW, et al. Effects of aerobic and/or resistance training on body mass and fat mass in overweight or obese adults. J Appl Physiol (1985). 2012 Dec;113(12): 1831-7.

26 Wilson JD. The measurement of the exchangeable pools of cholesterol in the baboon. J Clin Invest. 1970 Apr; 49(4):655-65.

27 Gómez-Ambrosi J, Salvador J, Páramo JA, Orbe J, de Irala J, Diez-Caballero A, et al. Involvement of leptin in the association between percentage of body fat and cardiovascular risk factors. Clin Biochem. 2002 Jun;35(4): 315-20.

28 Frühbeck G, Gómez-Ambrosi J, Salvador J. Leptin-induced lipolysis opposes the tonic inhibition of endogenous adenosine in white adipocytes. FASEB J. $2001 \mathrm{Feb} ; 15(2): 333-40$.

29 Hills M, Armitage P. The two-period cross-over clinical trial. Br J Clin Pharmacol. 1979 Jul;8(1):7-20. 